\title{
Space Shuttle Communications Coverage Analysis for Thermal Tile Inspection
}

\author{
Dr. Shian U. Hwu ${ }^{1}$ \\ Barrios Technology Inc., Houston, TX 77058 \\ Matthew Upanavage ${ }^{2}$ \\ ERC Inc., Houston, TX 77058 \\ John P. Boster ${ }^{3}$ \\ Jacobs Sverdrup, Houston, TX 77058 \\ Quin D. Kroll ${ }^{4}$ \\ NASA Johnson Space Center, Houston, TX 77058 \\ Mark A. Chavez ${ }^{5}$ \\ NASA Johnson Space Center, Houston, TX 77058
}

\begin{abstract}
The space shuttle ultra-high frequency Space-to-Space Communication System has to provide adequate communication coverage for astronauts who are performing thermal tile inspection and repair on the underside of the space shuttle orbiter (SSO). Careful planning and quantitative assessment are necessary to ensure successful system operations and mission safety in this work environment. This study assesses communication systems performance for astronauts who are working in the underside, non-line-of-sight shadow region on the space shuttle. All of the space shuttle and International Space Station (ISS) transmitting antennas are blocked by the SSO structure. To ensure communication coverage at planned inspection worksites, the signal strength and link margin between the SSO/ISS antennas and the extravehicular activity astronauts, whose line-of-sight is blocked by vehicle structure, was analyzed. Investigations were performed using rigorous computational electromagnetic modeling techniques. Signal strength was obtained by computing the reflected and diffracted fields along the signal propagation paths between transmitting and receiving antennas. Radio frequency (RF) coverage was determined for thermal tile inspection and repair missions using the results of this computation. Analysis results from this paper are important in formulating the limits on reliable communication range and $\mathrm{RF}$ coverage at planned underside inspection and repair worksites.
\end{abstract}

\section{Introduction}

$\mathrm{T}$ HE space shuttle orbiter (SSO) payload bay (PLB) ultra-high frequency (UHF) antenna provides communication links for extravehicular activity (EVA) astronauts who are performing thermal tile inspection and repair on the underside of the space shuttle. Careful planning and quantitative assessment are necessary to ensure successful system operations and mission safety for this underside environment. The PLB antenna, which is located inside the PLB, is blocked by the SSO structure, thereby affecting communications. Signals have to overcome this vehicle structure blockage to reach an astronaut at the underside worksite (Figures 1 and 2). To ensure that the RF coverage can meet requirements, the link margin evaluation must take into account the

\footnotetext{
${ }^{1}$ Sr. Engineering Specialist, Avionics Systems Analysis Section, JE-6WA, and AIAA Senior Member.

${ }^{2}$ Engineer, Avionics Systems Analysis Section, JE-6WA.

${ }^{3}$ Project Lead, Avionics Systems Analysis Section, JE-6WA.

${ }^{4}$ Communication Systems Simulation Lab Manager, Systems Evaluation \& Verification Branch, EV7.

${ }^{5}$ Project Engineer, Electromagnetic Systems Branch, EV4.
} 
signal attenuation that is due to vehicle blockage. The system must be able to compensate for signal attenuation, and provide adequate signal level and link margin throughout the designated area for the communication system to function effectively.

In shadow regions, non-line-of-sight (NLOS) locations will experience high path loss and signal degradation. This study is based on rigorous electromagnetic modeling, which takes into account diffractions along propagation paths over and around blockage structures. In many cases, as predicted by computer simulations and supported by experimental measurements, diffractions can result in adequate received signals and link margin for communication coverage in the NLOS shadow area. However, due to severe structure blockage and destructive interference, communications may not be available in some areas.

The purpose of this study is to assess the signal strengths and RF coverage performance for astronauts who are working in the shadow region underneath the space shuttle. Due to the large size of the shuttle, full-scale vehicle mockup measurements that require a large outdoor range are difficult to implement, which makes scaled vehicle mockup measurements in the NASA Johnson Space Center (JSC) Anechoic Chamber practical. Another approach in evaluating communication performance that is impacted by vehicle blockage is to use an electromagnetic modeling technique. Computer simulations are advantageous when (1) it is too expensive and dangerous to perform tests or experiments on a large, complex vehicle; and (2) the vehicle is unavailable or cannot be tested for a scenario.

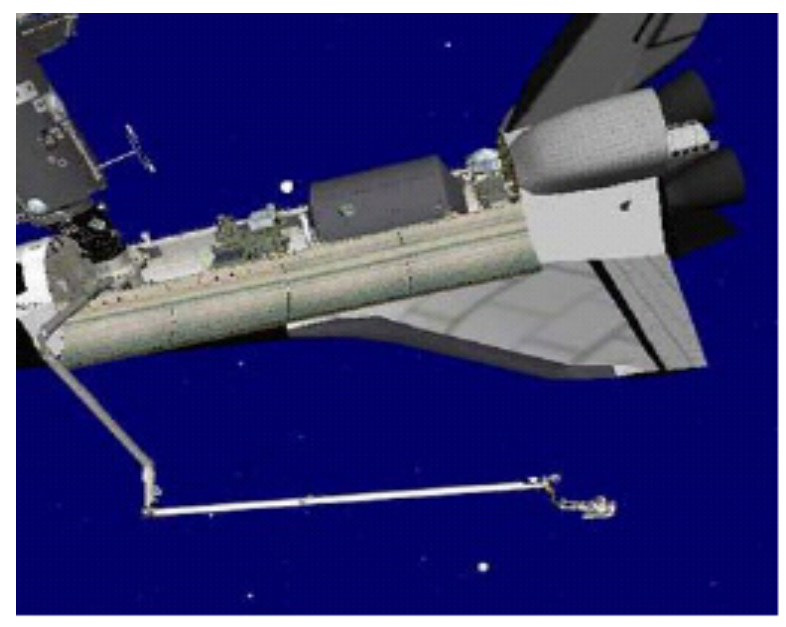

Figure 1. Astronaut transitioning to space shuttle underside worksite for thermal tile inspection.

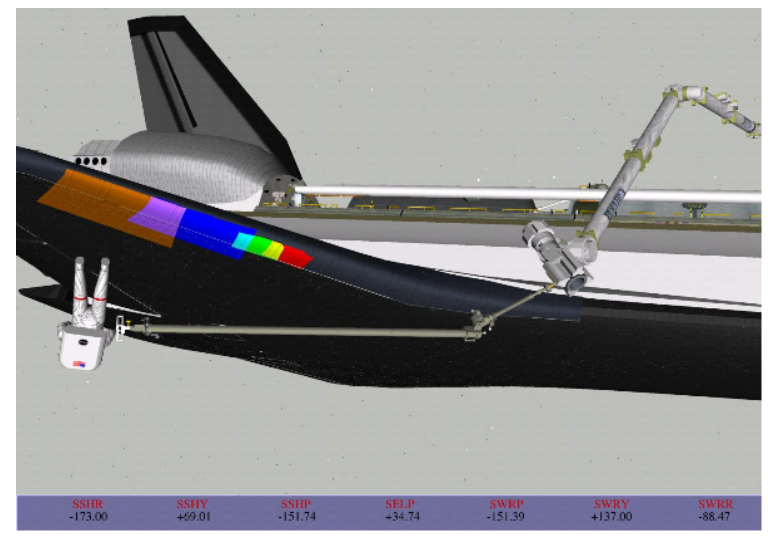

Figure 2. Astronaut performing thermal tile inspection and repair in worksite underneath the space shuttle.

In this study, the signal strength was obtained by computing the reflected and diffracted fields along the signal propagation paths between the transmitting and receiving antennas. Radio frequency $(\mathrm{RF})$ coverage was determined to be best for thermal tile inspection and repair missions based on the results that were obtained. Analysis results from this paper are important in determining underside communication performance analysis limits on reliable communication range and RF coverage at planned underside inspection and repair worksites.

\section{Computational Method}

The Geometrical Theory of Diffractions (GTD) was used in the simulations to account for the multipath and blockage effects from space shuttle structures.

At high frequencies, the scattering fields depend on the electrical and geometrical properties of the scatterer in the immediate neighborhood of the point of reflection and diffraction. In the field computation, incident, reflected, and diffracted fields are determined by the field incident on the reflection or diffraction point multiplied by a dyadic reflection or diffraction coefficient, a spreading factor, and a phase term. The reflected and diffracted field at a field point r', $\boldsymbol{E}^{\mathrm{r}, \mathrm{d}}\left(\mathrm{r}^{\prime}\right)$, in general has the following form: 


$$
\boldsymbol{E}^{\mathrm{r}, \mathrm{d}}\left(\mathrm{r}^{\mathrm{\prime}}\right)=\boldsymbol{E}^{\mathrm{i}}(\mathrm{r}) \boldsymbol{D}^{\mathrm{r}, \mathrm{d}} \boldsymbol{A}^{\mathrm{r}, \mathrm{d}}(\mathrm{s}) \mathrm{e}^{-\mathrm{jks}}
$$

where $\boldsymbol{E}^{\mathrm{i}}(\mathrm{r})$ is the field incident on the reflection or diffraction point $\mathrm{r}, \boldsymbol{D}^{\mathrm{r}, \mathrm{d}}$ is a dyadic reflection or diffraction coefficient, $\boldsymbol{A}^{\mathrm{r}, \mathrm{d}}(\mathrm{s})$ is a spreading factor, and $\mathrm{s}$ is the distance from the reflection or diffraction point $\mathrm{r}$ to the field point r'. $\boldsymbol{D}^{\mathrm{r}, \mathrm{d}}$ and $\boldsymbol{A}^{\mathrm{r}, \mathrm{d}}$ can be found from the geometry of the structure at reflection or diffraction point $\mathrm{r}$ and the properties of the incident wave there. Thus, the total fields $\left(\boldsymbol{E}^{\text {tot }}\right)$ can be obtained by summing the individual contributions of the direct field $\left(\boldsymbol{E}^{\text {dir }}\right)$, reflected field $\left(\boldsymbol{E}^{\text {ref }}\right)$, and diffracted field $\left(\boldsymbol{E}^{\text {dif }}\right)$ along the propagation paths as follows and also as shown in Figure 3:

$$
E^{t o t}=E^{d i r}+\sum_{n=1}^{N} E_{n}^{r e f}+\sum_{m=1}^{M} E_{m}^{d i f} \cdot(2)
$$

$\boldsymbol{E}^{t o t}=$ Total field at the observation point

$\boldsymbol{E}^{\text {dir }}=$ Direct fields from antennas

$\boldsymbol{E}^{r e f}=$ Reflected fields from plates and curve surfaces

$\boldsymbol{E}^{d i f}=$ Diffracted fields from plates and curve surfaces

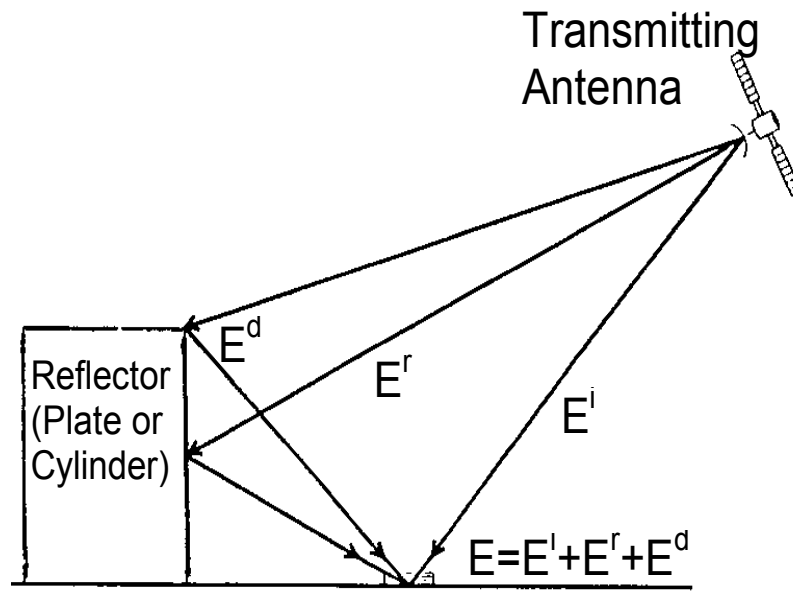

Figure 3. The GTD field computation.

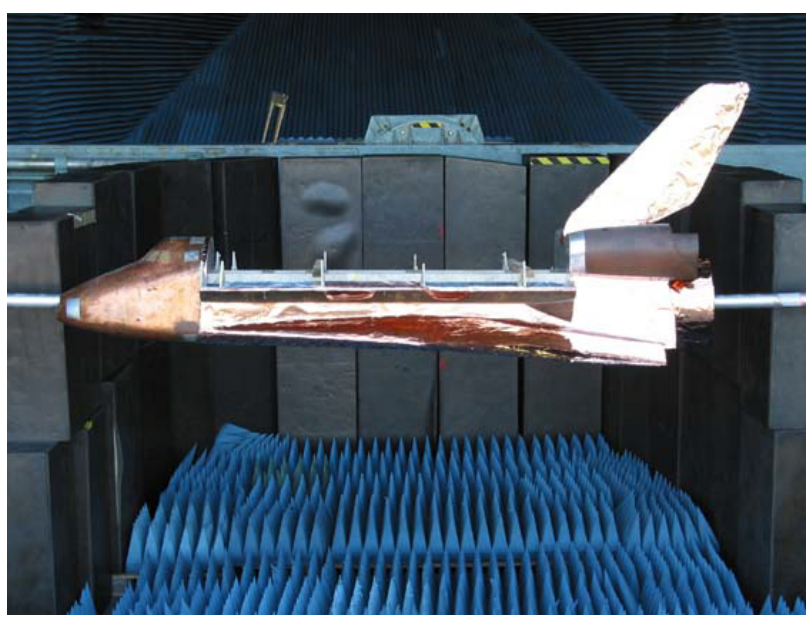

Figure 4. NASA JSC Anechoic Chamber nearfield measurements setup.

\section{Communication Performance Evaluation}

Communication performance is evaluated for underside communications when astronauts perform vehicle inspection and repair tasks. Data that were obtained from the computer simulations were validated with the Anechoic Chamber near-field measurement data.

Signal strengths that are underneath the space shuttle, which use the International Space Station (ISS) and space shuttle UHF antennas, were computed using the GTD simulation tool. The ISS antennas are available for underside repair missions when the space shuttle is docked to the ISS. For other shuttle-alone missions, only the space shuttle PLB antenna is available for underside inspection. Computed electric fields were compared to the signal strength corresponding to the 0 -dB link margin. The computational model was verified with test data [9]. Experimental data were obtained from the NASA JSC Anechoic Chamber near-field measurements. The test setup is shown in Figure 4. Reasonable agreement between measured and computed results is thus obtained $[10,11]$.

\section{A. Shuttle-Alone Missions}

The space shuttle PLB antenna is located deep in the shadow for belly communications, as shown in Figure 5. Underside coverage is expected to be poor due to severe vehicle structure blockage. 
The space shuttle PLB UHF antenna signal strengths at 1 and 2 meters underneath the SSO are shown in Figures 6 and 7. The signal level on the starboard side is higher than that on the port side due to the PLB UHF antenna location. Data indicate that due to a significant wing blockage in those areas, the lowest signal levels are in the regions near the tail. Signal levels improve slightly with increasing separation distance between the astronauts and the orbiter underside. To obtain optimum communication performance, an EVA astronaut may need to maneuver a distance of 0.3 or 0.6 meter, or adjust to a slightly different orientation of 10 or 20 degrees may be necessary. With the space shuttle alone, the PLB antenna can only provide marginal coverage over the forward half of the underside region. The underside aft region will therefore have bad coverage.

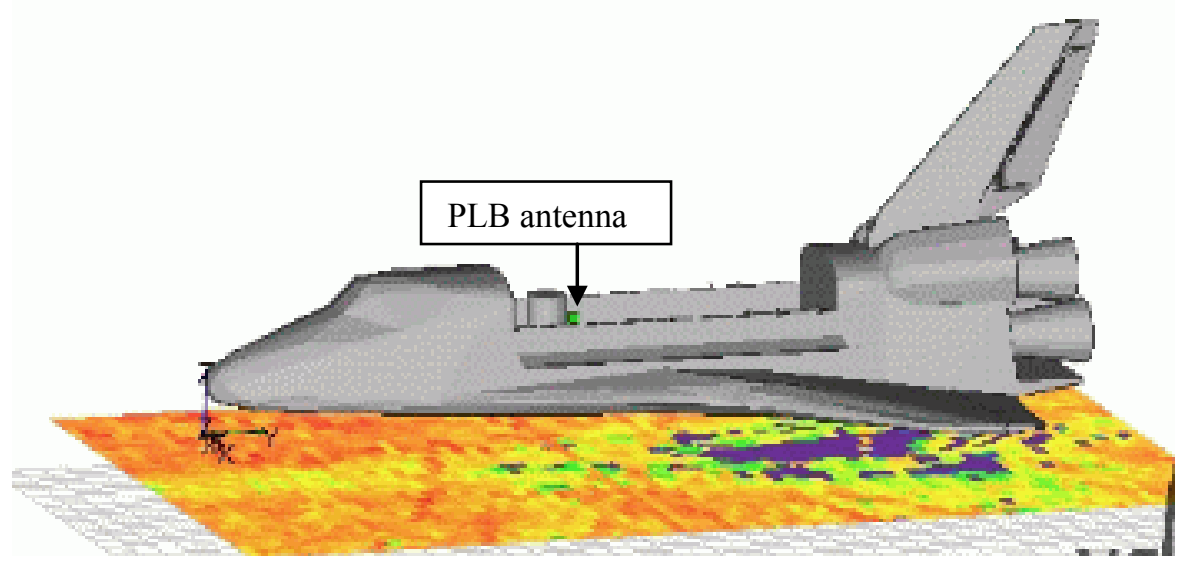

Figure 5. Space shuttle PLB UHF antenna location.
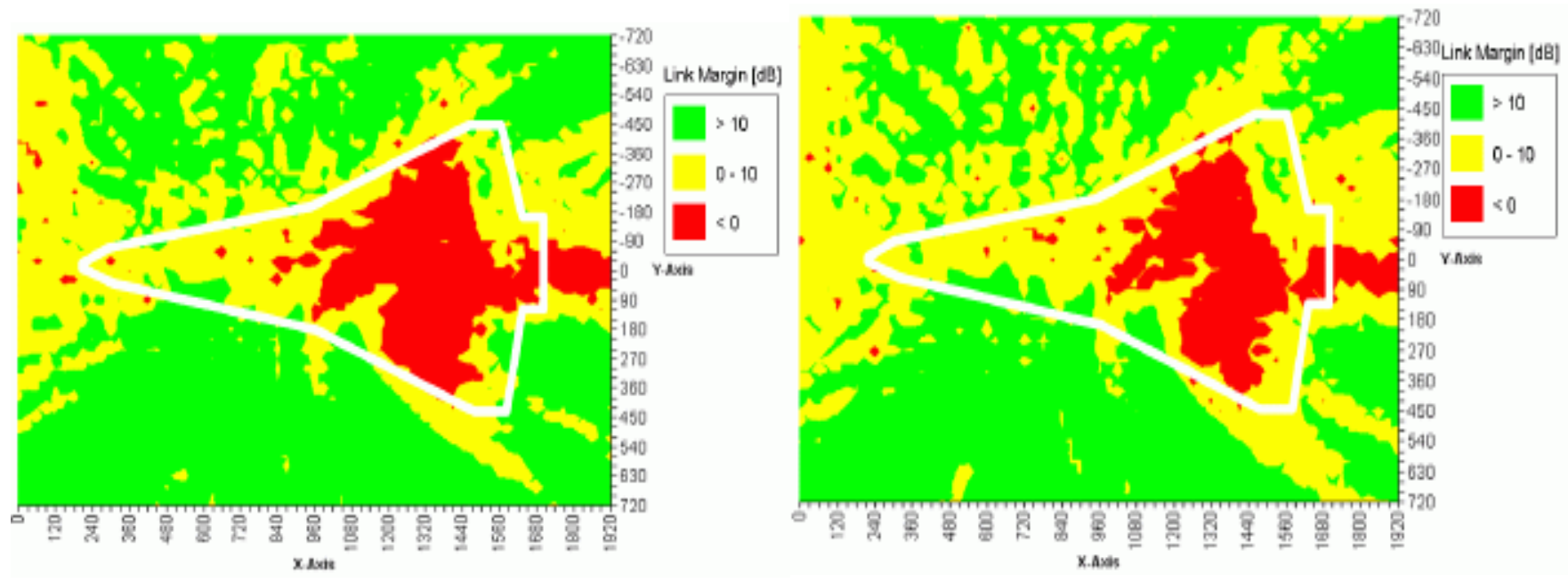

Figure 6. Space shuttle PLB UHF antenna signal strengths in terms of link margin $(\mathrm{dB})$ at 1 meter underneath the space shuttle.

Figure 7. Space shuttle PLB UHF antenna signal strengths in terms of link margin (dB) at 2 meters underneath the space shuttle.

\section{B. Space Shuttle Docked to ISS}

For the missions in which the space shuttle is docked to the ISS, the UHF system on both the space shuttle and ISS can be used to maximize the communication coverage. Computer simulations were performed using the ISS UHF Space-to-Space Communication System (SSCS) Lab and P1 truss antennas. There are four antennas total in the ISS SSCS system, two on each boom with one pointed foreward and one pointed aft. The ISS UHF antenna 
locations are shown in Figures 8 and 9. Since the space shuttle is docked at the foreward end of the ISS, the aft pointed SSCS antennas will have minimum contribution on the shuttle underside communications. Only forward pointed SSCS antennas will be analyzed here. These antennas may be used to improve coverage for shuttle underside communications.

The space shuttle PLB antenna is located deep in the shadow for belly communications. The ISS P1 truss and Lab antennas have less severe blockages than the space shuttle PLB antenna. As a result, the ISS UHF antennas should provide better coverage when the space shuttle is docked to the ISS.

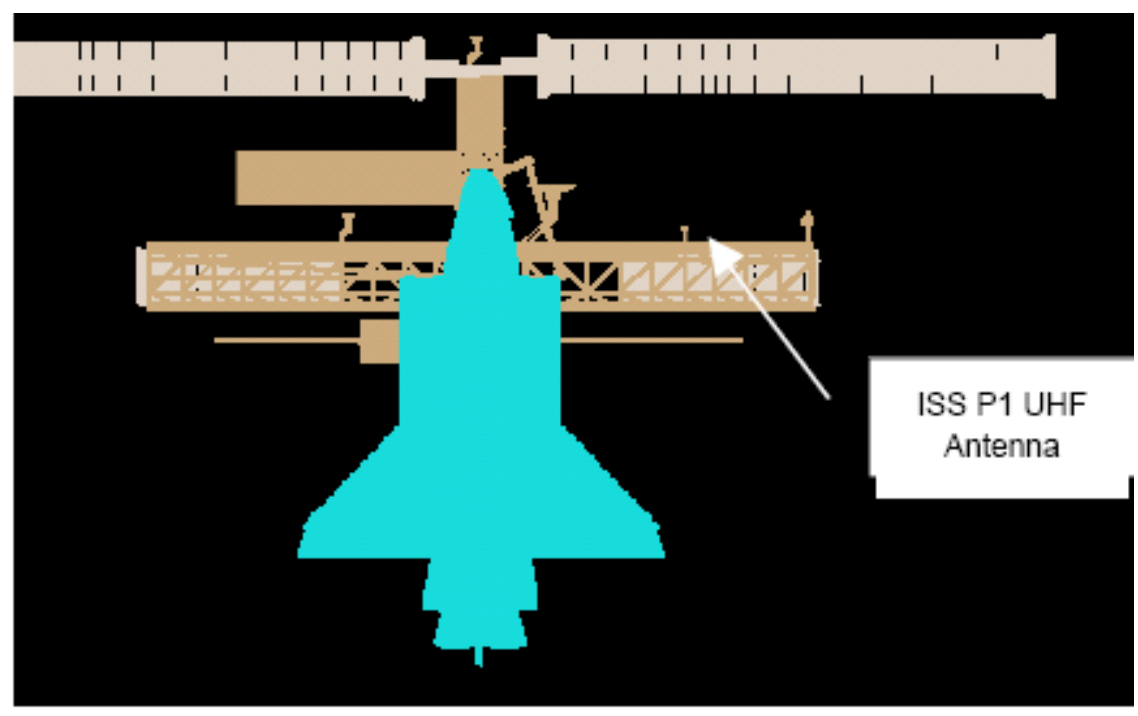

Figure 8. ISS UHF P1 truss antenna location.

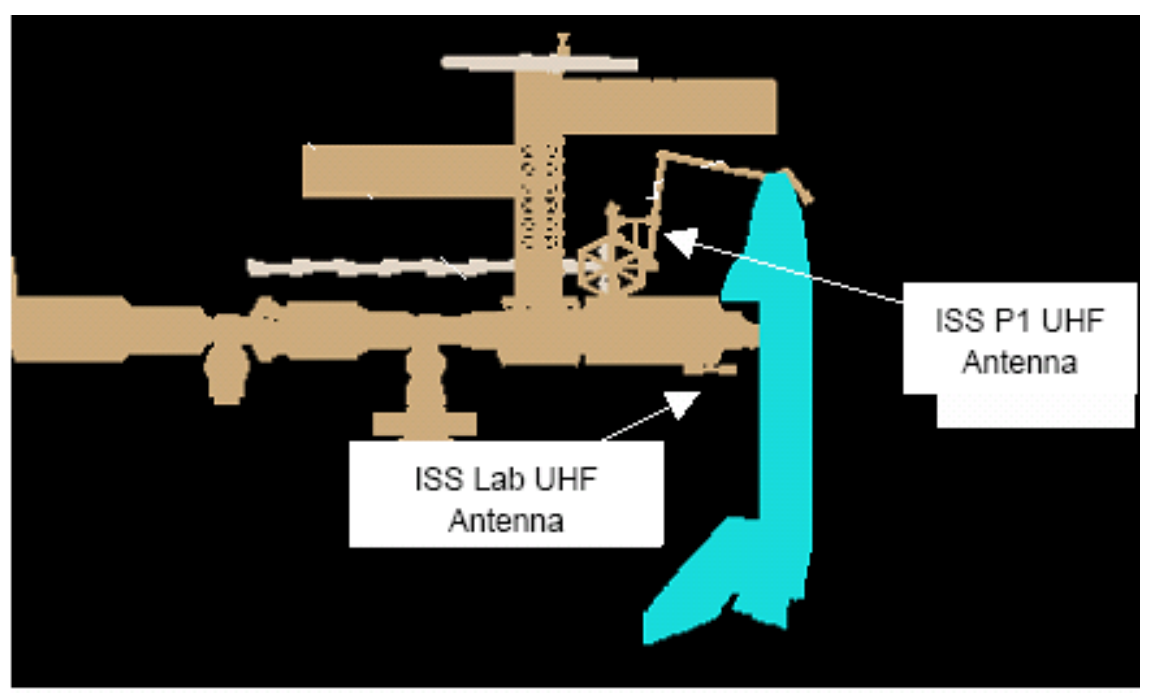

Figure 9. ISS UHF Lab antenna location.

Although both P1 truss and Lab antennas show degraded coverage areas near the center and tail section of the space shuttle belly, switching between the ISS P1 and Lab antennas will greatly improve coverage on the space shuttle belly, as shown in Figures 10 and 11. (This is actually accomplished by switching between two independent radio systems that are connected to each antenna.) Positive link margin is available at most of the underside area except at a few isolated spots. For space shuttle starboard-side inspection/repair, the ISS Lab antenna should be used 
as the primary antenna, and the P1 truss antenna as the secondary antenna. For space shuttle port-side inspection/repair, the ISS P1 truss antenna should be used as the primary antenna, and the Lab antenna as the secondary antenna. The primary antenna should be used until communication fails, at which time a switch should be made to the secondary antenna.

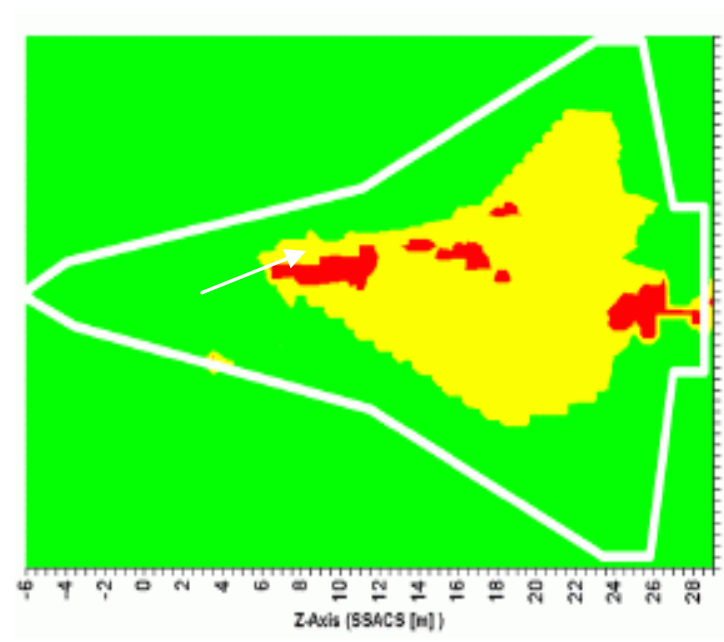

Figure 10. ISS combined UHF antenna signal strengths in terms of link margin $(\mathrm{dB})$ at 1 meter underneath the space shuttle.

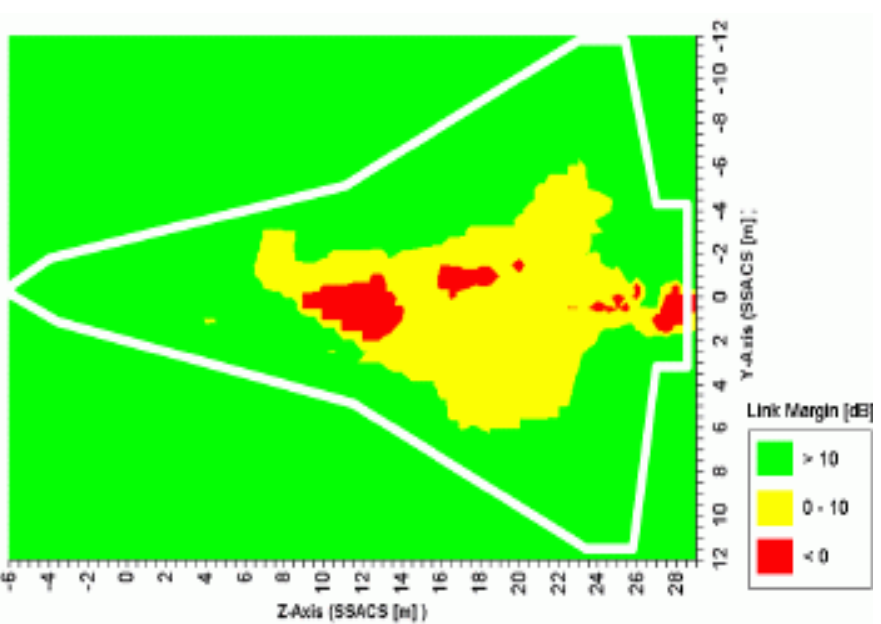

Figure 11. ISS combined UHF antenna signal strengths in terms of link margin (dB) at 2 meters underneath the space shuttle.

\section{Methods to Improve Underside Coverage}

Underside coverage is poor when using the space shuttle PLB antenna for shuttle-alone missions. Options to improve the underside communication coverage performance are discussed in this section.

\section{A. Relay Astronaut}

Underside communication coverage can be improved by placing an astronaut in a position in which to act as a relay. The radio is not repeating the RF transmission. The relay astronaut is verbally repeating the words to the second astronaut in the underside communication blockage area. The signal attenuation due to blockage is greatly reduced by such a relay operation. With an appropriate placement, a relay astronaut can change underside operations from NLOS to line-of-sight (LOS). To be effective, the relay astronaut should be placed on the starboard side of the space shuttle, forward of the PLB doors.

A direct LOS from the underside astronaut to the relay astronaut will provide an operational work-around to the vehicle blockage of the communication signals. The relay astronaut is at a location where a good link margin of 10 $\mathrm{dB}$ or better to the PLB antenna is expected. The suggested relay astronaut location is shown in the greenhighlighted area in Figure 12, where communication links are expected to be good.

\section{B. Underside Air Traffic Control Antenna}

Underside communication coverage can be improved by using the very-high frequency (VHF) Air Traffic Control (ATC) voice antenna that is located on the underside of the space shuttle, as shown in Figure 13. The ATC antenna would be connected to the PLB transmit port, and the PLB antenna would be connected to the airlock transmit port, as shown in Figure 14. The airlock port transmission power is $30 \mathrm{~dB}$ below that of the PLB port. The RF link inside the airlock would be lost. The PLB antenna would have a reduction in communications range due to the $30-\mathrm{dB}$ attenuator in the airlock port. This change in configuration would be performed by the astronauts while they are in orbit, but only if required during an emergency situation.

The ATC antenna was originally designed for VHF, not UHF, operations. As a result, the ATC antenna will have degraded performance for operation at UHF. Measurements were carried out on the ATC antenna to determine the 
antenna radiation patterns and mismatch loss for out-of-band usage at UHF. Test data indicate that the peak antenna gain is degraded by $7 \mathrm{~dB}$ and the mismatch loss is increased by $4.3 \mathrm{~dB}$ at UHF.

Improvement to underside communication performance from the usage of the ATC antenna is significant even with a degraded antenna. The ATC antenna changes underside operations from NLOS to LOS.

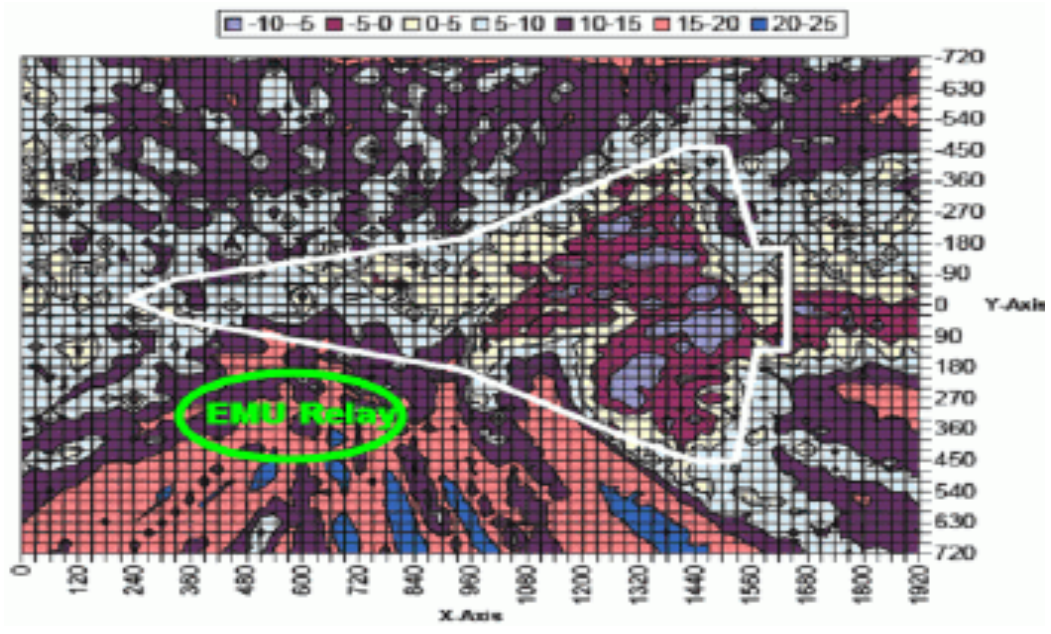

Figure 12. The suggested relay astronaut location is shown in the green-highlighted area.

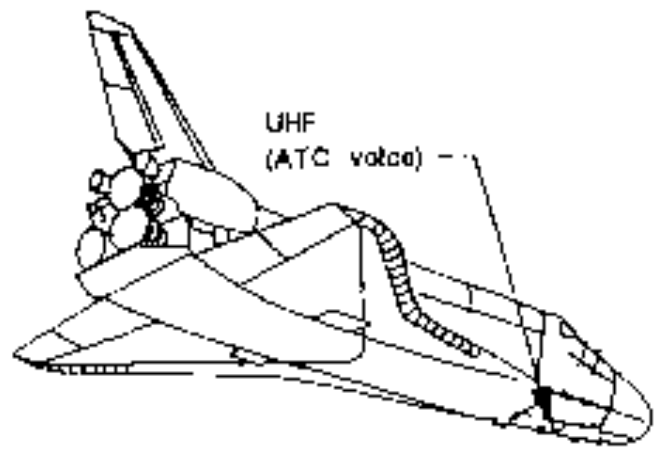

Figure 13. The UHF ATC voice antenna on the underside can be an alternative to improve underside communication coverage.

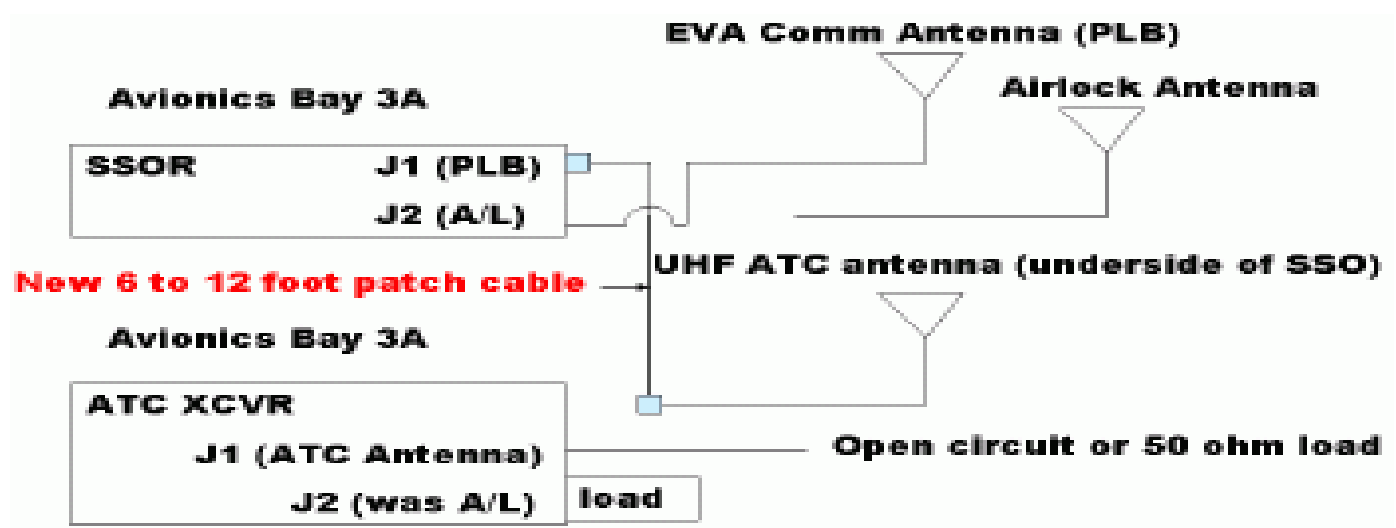

Figure 14. An extension cable to connect the SSO radio to the UHF ATC antenna on the underside improves underside communication coverage. 


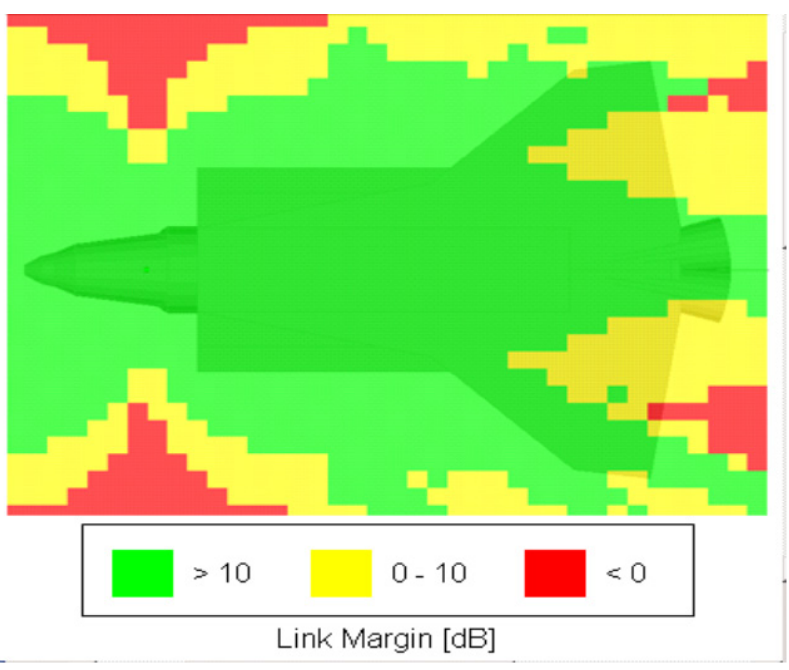

Figure 15. The space shuttle ATC antenna provides good coverage for underside work.

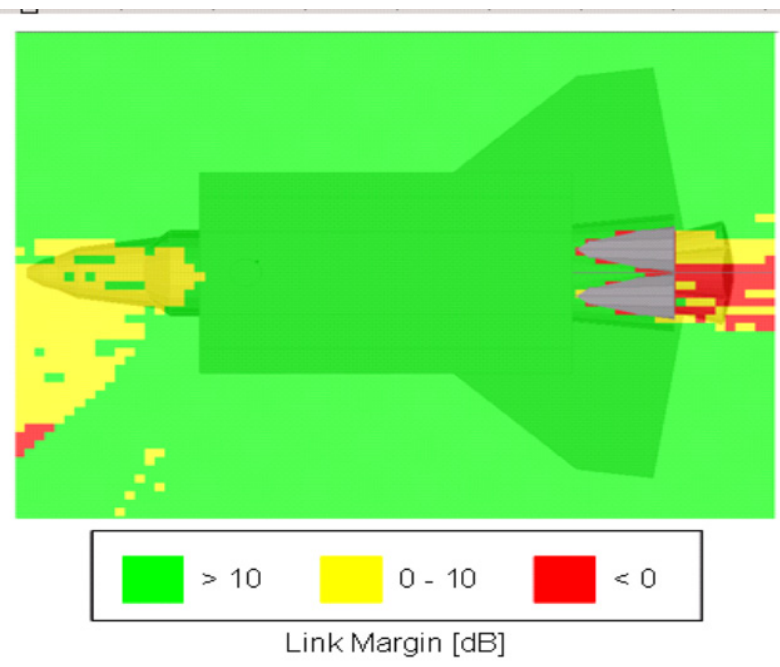

Figure 16. The space shuttle PLB antenna provides good coverage throughout the PLB area.

The ATC antenna is expected to have good coverage 1 meter from the SSO belly. As shown in Figure 15, the link margin is improved significantly in most underside worksites. Marginal coverage occurs toward the aft sections of the wings. An area of bad coverage that is lateral to the crew cabin will not affect underside worksite communications. The space shuttle PLB antenna is expected to have bad coverage 1 meter from the space shuttle belly. Previous analysis shows poor coverage for nominal space shuttle PLB antenna configuration.

The space shuttle PLB antenna is expected to have good coverage throughout the PLB, as shown in Figure 16. The close proximity of the PLB antenna to the orbiter docking system causes marginal coverage over the forward area of the space shuttle. The ATC antenna is expected to have bad coverage throughout the PLB. Using the PLB transmitter port to power the ATC antenna provides extended range and improved coverage on the underside of the space shuttle.

Simulation results indicate that using the airlock transmitter port to power the PLB antenna results in adequate coverage in the PLB. Coverage along the sides of the space shuttle is good. However, orbiter-to-orbiter coverage may be reduced if the PLB antenna is connected to the airlock port, which has reduced power. This PLB-to-PLB antenna communication scenario would only be used for a emergency rescue mission with another orbiter. The rescue orbiter would have full power, but the modified orbiter would have reduced power.

\section{Conclusion}

In this study, the communication performance underneath the SSO using the space shuttle and ISS UHF antennas was analyzed. Shadowing effects from the space shuttle structures blocking the UHF antennas were investigated. A computer simulation tool that is based on the GTD method was used to compute signal strengths. RF coverage areas for the underside communications were determined based on the results that were obtained in this study.

Good EVA astronaut communication coverage can be achieved by using the ISS Lab antenna as the primary antenna and switching to the ISS P1 truss antenna when necessary. Signal levels improve slightly with increasing separation distance between the EVA astronauts and the orbiter underside. Data indicate that the lowest signal levels are in the regions near the tail due to significant wing blockage in those areas. The signal level is, in general, better for the ISS Lab and P1 antennas than for the space shuttle PLB antenna due to the favorable location and pointing directionof the ISS Lab/P1 antenna. The ISS Lab antenna provides better signal levels on the shuttle port side, and the ISS P1 antenna provides better signal levels on the shuttle starboard side.

Underside coverage is poor when using the space shuttle PLB antenna for shuttle-alone missions. Options to improve underside communication coverage performance were investigated. An astronaut can possibly improve a poor link condition by a reorientation. An astronaut relay scenario that uses a second astronaut to relay messages can also improve the coverage area. An astronaut who is placed on the space shuttle starboard side near the SSO belly 
will be able to relay voice to/from the astronaut who is working on the tile inspection under the belly of the space shuttle.

A feasible option in an emergency situation to improve underside communications is to use the PLB transmitter port to power the ATC antenna. This alternative configuration, which will necessitate minimum hardware modifications that will be performed by astronauts, provides extended range and improved coverage of the space shuttle underside. Improved underside communication performance from usage of the ATC antenna is significant even with a degraded ATC antenna. The ATC antenna changes underside operations from NLOS to LOS. Simulation results indicate that using the airlock transmitter port to power the PLB antenna results in adequate coverage in the PLB. Coverage along the sides of the space shuttle is also good.

\section{Acknowledgment}

The study described in this paper was carried out under contracts with the NASA Johnson Space Center.

\section{References}

${ }^{1}$ R. G. Kouyoumjian and P. H. Pathak, "A uniform geometrical theory of diffraction for an edge in a perfectly conducting surface," Proceedings of the IEEE, vol. 62, no. 11, pp. 1448-1461, Nov. 1974.

${ }^{2}$ R. J. Marhefka and W. D. Burnside, "Antennas on complex platforms," Proceedings of the IEEE, vol. 80, no. 1, pp. 204-208, Jan. 1992.

${ }^{3}$ W. Burnside, M. Gilreath, R. Marhefka, and Y. Chong, "A study of KC-135 aircraft antenna patterns," IEEE Trans. Antennas and Propagation, [legacy, pre-1988], vol. 23, no. 3, pp. 309-316, May 1975.

${ }^{4}$ R. J. Marhefka, "The other NEC (BSC) an asymptotic complement," Antennas and Propagation Society Symposium, 2004. IEEE, vol. 3, pp. 2911-2914, 20-25 June 2004.

${ }^{5}$ R. J. Marhefka and J. W. Silvestro, "Near zone - basic scattering code user's manual with space station applications," NASA CR-181944, Dec. 1989.

${ }^{6}$ S. Hwu, J. Dobbins, Y. Loh, C. Sham, and Q. Kroll, "Space Shuttle UHF Communications Performance Evaluation,” IEEE Aerospace and Electronic Systems Magazine, vol. 20, pp. 9-14, Oct. 2005.

${ }^{7}$ XGTD User's Manual, Remcom Inc., 2007.

${ }^{8}$ J. A. Dobbins, "Engineering Evaluation of 1/10th Scale Shuttle Payload Bay Antennas in 1/10th Scale Orbiter Mockup," TPS no. 610420032, EV4, NASA Johnson Space Center, July 2004.

${ }^{9}$ S. Hwu, "Space Shuttle Orbiter payload bay UHF SSOR and WVS antenna test data coverage analysis," NASA Johnson Space Center, EV7-04-3058, Sep. 2004.

${ }^{10}$ S. Hwu, J. Boster, and T. Z. Abebe, "Space Underside Communications Performance Evaluation," NASA Johnson Space Center, EV7-05-3104, Dec. 2004.

${ }^{11}$ S. U. Hwu and T. Z. Abebe, "Space Shuttle Orbiter Payload Bay SSOR and WVS UHF Antenna RF Coverage Requirements Analysis," NASA Johnson Space Center, EV7-05-3103, Nov. 2004. 